\title{
ADAPTIVE ROUNDING OPERATOR FOR EFFICIENT WYNER-ZIV VIDEO CODING
}

\author{
Jeffrey J. Micallef, Reuben A. Farrugia and Carl J. Debono \\ Department of Communication and Computer Engineering, University of Malta, Msida, Malta \\ jeffrey.micallef@ieee.org,reuben.farrugia@um.edu.mt, c.debono@iee.org
}

\begin{abstract}
The Distributed Video Coding (DVC) paradigm can theoretically reach the same coding efficiencies of predictive block-based video coding schemes, like H.264/AVC. However, current DVC architectures are still far from this ideal performance. This is mainly attributed to inaccuracies in the Side Information (SI) predicted at the decoder. The work in this paper presents a coding scheme which tries to avoid mismatch in the SI predictions caused by small variations in light intensity. Using the appropriate rounding operator for every coefficient, the proposed method significantly reduces the correlation noise between the Wyner-Ziv (WZ) frame and the corresponding SI, achieving higher coding efficiencies. Experimental results demonstrate that the average Peak Signal-to-Noise Ratio (PSNR) is improved by up to $0.56 \mathrm{~dB}$ relative to the DISCOVER codec.
\end{abstract}

Index Terms - Adaptive quantization, correlation noise, distributed video coding, source representation, Wyner-Ziv coding.

\section{INTRODUCTION}

The traditional video coding schemes present an asymmetric distribution of complexities, with the encoder being orders of magnitude more complex than the decoder [1]. This asymmetry is not suitable for applications that necessitate low complexity encoders, such as wireless devices and miniature endoscopic cameras. Distributed Video Coding (DVC) is a promising solution for such scenarios. It exploits the Wyner-Ziv (WZ) theorem [2], which shows that most of the computational burden involved in exploring the source statistics can be shifted from the encoder to the decoder without affecting the coding efficiency.

The DVC architectures predict the WZ frames at the decoder using Motion Compensated Temporal Interpolation (MCTI) between the adjacent key frames. These predictions, known as the Side Information (SI), are then used to aid compression. The decoder models the correlation noise between the WZ frame and the SI and feed the soft-input information into a Low-Density Parity-Check Accumulate (LDPCA) decoder. This will then request only a subset of parity information to recover the original bit-planes of $\mathrm{WZ}$ frame, achieving compression. Higher coding efficiencies are thus obtained when the accuracy of the soft-input values increases, reducing mismatch between the bit-planes predicted by the soft-input predictions and those of the original WZ frame.

The authors in [3]-[5] improved the correlation between the WZ frames and the SI using carefully selected codeword representations, such as gray codes. Meanwhile, previous work in [6] considered the discrepancy within the previously decoded bit-planes to predict some of the mismatch in SI and corrected them to improve correlation. Such methods improve compression without affecting the quality of the reconstructed video. Conversely, non-uniform quantizers which are optimally designed to reduce reconstruction errors were adopted in [7]-[8]. Similarly, the authors in [9]-[10] reduced quantization noise by considering uniform quantizers whose intervals can adapt with the characteristics of the transform coefficient band. Meanwhile, Jung and Karam transmitted only the parity bits required to correct the bit-planes that could provide an estimated high Rate-Distortion (R-D) performance [11]. Later on, the same authors used different quantization intervals for different parts of the WZ frame based on the local R-D characteristics [12].

Nevertheless, none of the above literature considers that the traditional DVC schemes can generate inaccurate softinput values when the coefficients, found near the endpoints of the quantization intervals, are affected by small variations in light intensity. These inaccuracies can degrade coding efficiency. To alleviate this, it is proposed that the encoder and decoder should distinguish between the floor and round operators during quantization. These operators consider a different quantization interval and can generate soft-input predictions with different accuracies. The proposed scheme should thus choose the function that is most likely to provide accurate soft-input values. Simulation results show that the proposed scheme can improve the overall Rate-Distortion performance by up to $0.56 \mathrm{~dB}$ relative to the DISCOVER codec, with minimal increase in the encoder's complexity.

This paper is organized as follows: the transform domain Wyner-Ziv video coding architecture used is discussed in Section 2. Section 3 briefly reviews how small variations in intensity can considerably reduce compression efficiency, whereas Section 4 discusses the coding scheme which can be used to choose the appropriate quantization function. Experimental results are then presented in Section 5 and Section 6 provides the final comments and conclusion. 


\section{WYNER-ZIV VIDEO CODING FRAMEWORK}

The Wyner-Ziv (WZ) video coding architecture adopted in this work is illustrated in Fig. 1 and it is based on the DISCOVER codec [13]-[14]. The incoming frames are divided into two groups, with the even frames encoded using WZ techniques and denoted as $\mathbf{W Z}_{2 \mathrm{x}}$. Conversely, the odd frames are encoded as key frames using H.264/AVC Intra coding scheme and denoted as $\mathbf{K}_{2 \mathrm{x}-1}$ and $\mathbf{K}_{2 \mathrm{x}+1}$ for the backwards and forwards key frames of the WZ frame. The WZ frames will first undergo a block-based Discrete Cosine Transform (DCT) and the resulting coefficient bands are uniformly quantized into $2^{L}$ levels using a dead-zone quantizer [9]. This can adapt with the dynamic range of the coefficient band and was set to use the appropriate quantization function, as indicated by the binary map $\mathbf{M}$ which is generated as described in Section 4. The quantized symbol stream $\overline{\mathbf{Q}}$ is then converted into Gray codes $\mathbf{Q}$ and the $b^{\text {th }}$ bit-plane $\mathbf{Q}^{(b)}$ is extracted using the Bit-plane Extraction module and fed into the LDPCA encoder [15]. The resulting syndrome bits are finally stored in a buffer to be transmitted incrementally as requested by the decoder.

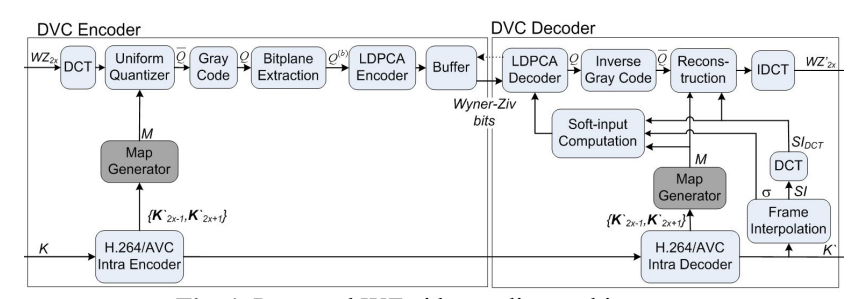

Fig. 1. Proposed WZ video coding architecture.

The decoder reconstructs the adjacent key frames $\mathbf{K}^{\prime}{ }_{2 \mathrm{x}-1}$ and $\mathbf{K}_{2 \mathrm{x}+1}$, and predicts the side information using MCTI techniques [16]. The binary map $\mathbf{M}$, used by the encoder, is then re-generated at the decoder since this is needed for the correct operation of both the Soft-input Computation and the Reconstruction modules. The correlation noise between the original WZ frame and the SI is subsequently modeled as a Laplacian distribution, whose $\sigma$ parameters are calculated at band level using the difference between the forwards and backwards motion compensated frames [17]. This model is used to generate the soft-input values from the SI, considering the previously decoded bit-planes [18]. These are then fed into the LDPCA decoder, which requests parity bits from the encoder to correct the initial prediction given by the soft-input predictions and recover the bit-planes of the WZ frame. All the decoded bit-planes are subsequently joined together and mapped into the quantized symbols $\overline{\mathbf{Q}}$ using inverse gray codes. The WZ coefficients are finally reconstructed using the quantized symbols $\overline{\mathbf{Q}}$ and the side information [19], and passed through an inverse DCT to get the WZ frame in the pixel domain.

\section{EFFECT OF VARIATIONS IN ILLUMINATION}

During reconstruction, the decoder truncates all the coefficients of the SI within the interval indicated by the WZ indices $\mathbf{\mathbf { Q }}$ [19], thus limiting the prediction errors to within a fixed range set by the quantization interval. The $\mathrm{WZ}$ indices $\overline{\mathbf{Q}}$ can be obtained efficiently at the decoder using channel coding techniques where the side information is used whilst generating the soft-input predictions to aid compression. Yet, it can be shown that the soft-input values predicted for the WZ coefficients near the endpoints of the quantization interval have a high probability of being incorrect.

For a WZ coefficient at position $\mathbf{p}=\left(p_{\mathrm{x}}, p_{\mathrm{y}}\right)$, denoted as $\mathbf{W Z}_{2 \mathrm{x}}(\mathbf{p})$, the soft-input prediction is calculated by modeling a Laplacian distribution around the corresponding SI coefficient $\mathbf{S I}_{2 \mathrm{x}}(\mathbf{p})$ and then sum the appropriate areas under the distribution as indicated by the previously decoded bitplanes [18]. Fig. 2 considers the DC coefficient band with a range $\left[0,2^{11}\right]$ quantized at 8 -levels using the floor operator (as in [13]-[14] and [16]) and Gray code representation. If a WZ DC coefficient $\mathbf{W Z}_{2 \mathrm{x}}(\mathbf{p})$ of 254 is predicted with $\mathbf{S I}_{2 \mathrm{x}}(\mathbf{p})$ of 259 due to variations in light intensity, the $3^{\text {rd }}$ bit-plane is incorrectly predicted as having a bit-value of 1 , since $P\left(\mathbf{Q}^{(3)}=1 \mid \mathbf{S I}, \mathbf{Q}^{(2)}, \mathbf{Q}^{(1)}\right)$ is greater than $P\left(\mathbf{Q}^{(3)}=0 \mid \mathbf{S I}, \mathbf{Q}^{(2)}, \mathbf{Q}^{(1)}\right)$ as shown in Fig. 2(a). Even though gray codes were adopted to improve correlation, it is observed that the soft-input values predicted for bit-plane $b$ is always incorrect when, due to small variations in light, the $\mathbf{W Z}_{2 \mathrm{x}}(\mathbf{p})$ and $\mathbf{S I}_{2 \mathrm{x}}(\mathbf{p})$ coefficients fall within adjacent intervals set for that bitplane. The $\mathrm{WZ}$ coefficients $\mathbf{W Z}_{2 \mathrm{x}}(\mathbf{p})$ near the endpoints of the quantization intervals indicated in Fig. 2(b), which coincide with the endpoints of all the intervals for $b \in\{1, \ldots$, $L\}$ will thus have a higher probability of mismatch. This is because the SI has a high probability of falling within the adjacent interval set for one of the bit-planes. Similarly, when using the round operator whose endpoints of the intervals are set as in Fig. 2(c), a lot of unreliable soft-input values are expected for DC coefficients near these endpoints.

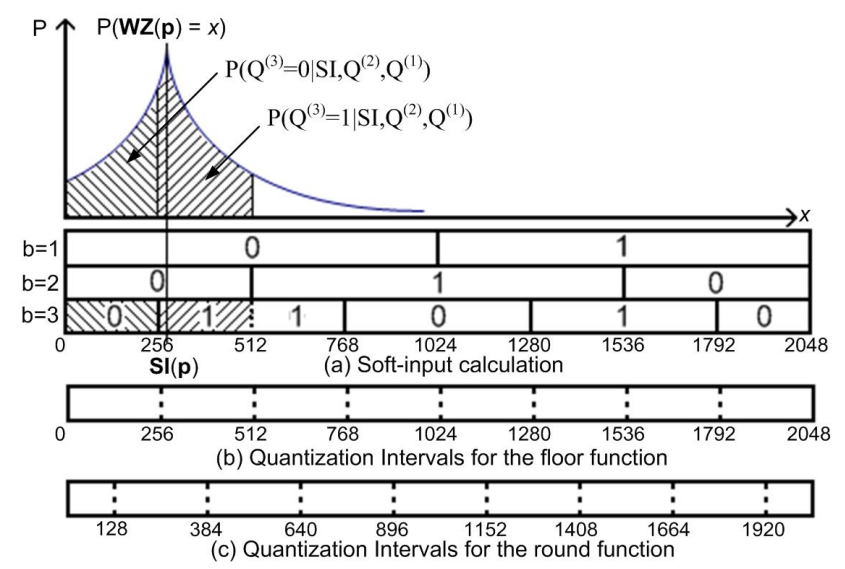

Fig. 2. Calculating Soft-input values with Gray code representation. 
Fig. 3(a) illustrates the level of correlation noise between the DC values of the $51^{\text {st }}$ frame in the Hall monitor sequence and its SI. Fig. 3(b)-(c) show the locations where the bit-planes of the WZ indices $\mathbf{Q}^{(b)}$ do not match the corresponding bit-plane predicted by the soft-input values $\mathbf{S}^{(b)}$ for one of the bit-planes $b \in\{1, \ldots, L\}$. Here, the coefficients were quantized at 64-levels using either the floor or the round operator. Clearly both operators provide a lot of mismatch in areas where the correlation noise is low. Similar observations were also made for all the other AC coefficients. These unreliable predictions can severely degrade compression efficiency, because the decoder needs a lot of parity information to recover the bit-planes of the WZ indices $\mathbf{Q}^{(b)}$ from the corresponding soft-input predictions. Furthermore, truncating the $\mathbf{S I}_{2 \mathrm{x}}(\mathbf{p})$ coefficient within the $\overline{\mathbf{Q}}$ interval during reconstruction will only result in a minimal enhancement in quality, since the side information offers a very good prediction of the $\mathrm{WZ}$ coefficient $\mathbf{W} \mathbf{Z}_{2 \mathrm{x}}(\mathbf{p})$.

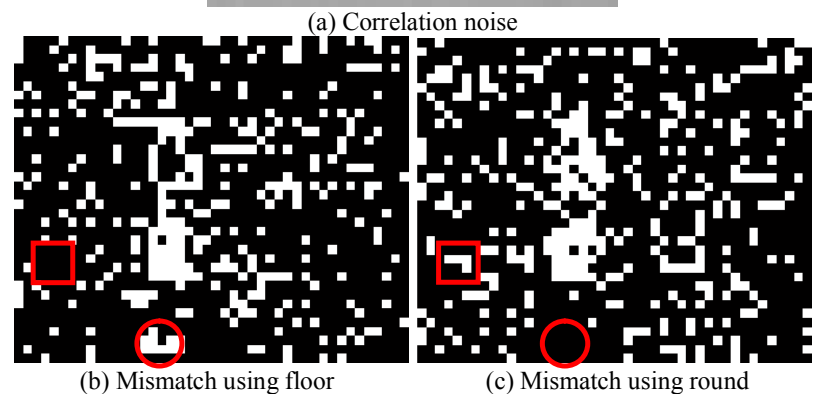

Fig. 3. Correlation noise and mismatch between bit-planes $\mathbf{Q}^{(\mathrm{b})}$ and $\mathbf{S}^{(\mathrm{b})}$.

\section{PROPOSED METHODOLOGY}

It is observed that the $\mathbf{W Z}_{2 \mathrm{x}}(\mathbf{p})$ coefficients enclosed within the circle in Fig. 3(b) are near the endpoints in Fig. 2(b) and thus the floor operator can result in poor soft-input values being predicted for one of the bit-planes. Yet, better soft-input values can be generated for such regions if the round operator is used as seen in Fig. 3(c). This is because, for all bit-planes $b \in\{1, \ldots, L\}$, these coefficients are further away from the endpoints in Fig. 2(c) and hence $\mathbf{W Z}_{2 \mathrm{x}}(\mathbf{p})$ and $\mathbf{S I}_{2 x}(\mathbf{p})$ coefficients have a higher probability of falling within the same interval, with more accurate soft-input values being generated. Similarly, the DC coefficients found within the square have more correct soft-input predictions when quantized with the floor operator rather than the round operator, since the coefficients are near the endpoints in Fig. 2(c). Therefore we propose to use an adaptive quantization scheme that chooses between the floor and round operator in order to improve the accuracy of the soft-input values for all bit-planes. This however produces a small loss in quality, caused by refraining to correct the coefficients with low prediction errors.

The best quantization operator to be considered for each coefficient location $\mathbf{p}$ is determined using the co-located coefficients of the reconstructed key frames $\mathbf{K}_{2 \mathrm{x}-1}{ }^{\mathrm{DCT}}(\mathbf{p})$ and $\mathbf{K}_{2 \mathrm{x}+1}{ }^{\mathrm{DCT}}(\mathbf{p})$. Unlike the WZ or the SI frames, these frames are readily available at both sides of the codec to maintain synchronization and they are highly correlated to both $\mathbf{W Z}_{2 \mathrm{x}}(\mathbf{p})$ and $\mathbf{S I}_{2 \mathrm{x}}(\mathbf{p})$ coefficients to predict their position relative to the endpoints in Figs. 2(b)-(c).

A series of offline experiments were carried out to determine the quantization operator to be chosen, given the co-located coefficient values of the adjacent key frames. During these experiments the key frames were quantized using the same quantization matrices used for the WZ frame, starting with the DC coefficients which have a range of [0, $\left.2^{11}\right)$ and are quantized at 128 -levels $(Q S=16)$. For every DC coefficient within the $\mathrm{WZ}$ frame $\mathbf{W Z}_{2 \mathrm{x}}(\mathbf{p})$, the bin indices of the adjacent key frames were then denoted as:

$$
i_{2 x-1}(p)=\left\lfloor\frac{\mathrm{K}_{2 \mathrm{x}-1}^{\prime D C T}(p)}{Q S}\right\rfloor, i_{2 x+1}(p)=\left\lfloor\frac{\mathrm{K}_{2 \mathrm{x}+1}^{D C T}(p)}{Q S}\right\rfloor
$$

where $Q S$ represents the width of the quantization intervals for the given band. The WZ frames were divided into three regions and the probability of mismatch studied as follows:

Region 1 considers the locations where the coefficients of the adjacent key frames are within the same interval i.e. $\boldsymbol{i}_{2 x-1}(\mathbf{p})=\boldsymbol{i}_{2 x+l}(\mathbf{p})$. For such regions of low motion, the differences $\mathbf{D}_{2 x-1}(\mathbf{p})$ and $\mathbf{D}_{2 x+l}(\mathbf{p})$, between the coefficient $\mathbf{K}_{2 \mathrm{x}-1}, \mathbf{p C T}^{\mathrm{DCT}}$ and $\mathbf{K}_{2 \mathrm{x}+1}, \mathbf{p C T}$ ), and the lower endpoint of the quantization intervals in which they are found, were established using:

$$
\begin{gathered}
\mathbf{D}_{2 x-1}(\mathbf{p})=\mathbf{K}_{2 \mathrm{x}-1}, \underset{\mathrm{DCT}}{\mathrm{DC}}-\boldsymbol{i}_{2 x-1}(\mathbf{p}) \times Q S \\
\mathbf{D}_{2 x+1}(\mathbf{p})=\mathbf{K}_{2 \mathrm{x}+1}^{,}, \underset{\mathrm{DCT}}{\mathrm{p})-\boldsymbol{i}_{2 x+1}(\mathbf{p}) \times Q S}
\end{gathered}
$$

The locations where, at least, one of the bit-planes $b \in\{1, \ldots, L\}$ of the WZ indices $\mathbf{Q}^{(b)}$ does not match the corresponding bit-planes predicted by the sign of the softinput values $\mathbf{S}^{(b)}$, were considered. These were then used to accumulate the probability of mismatch for every possible combination of $\mathbf{D}_{2 x-1}(\mathbf{p})$ and $\mathbf{D}_{2 x+1}(\mathbf{p})$ over the Hall monitor and Foreman sequences. Fig. 4(a)-(b) show the resulting statistics, with the columns representing $\mathbf{D}_{2 x-1}(\mathbf{p})$ ranging from 0 to $Q S$-1, whilst the rows represent $\mathbf{D}_{2 x+1}(\mathbf{p})$ within the same range. The cells represent the corresponding probability of mismatch obtained when using (a) the floor or (b) the round operator, considering a lighter shade of grey for cells having a higher probability of mismatch. 


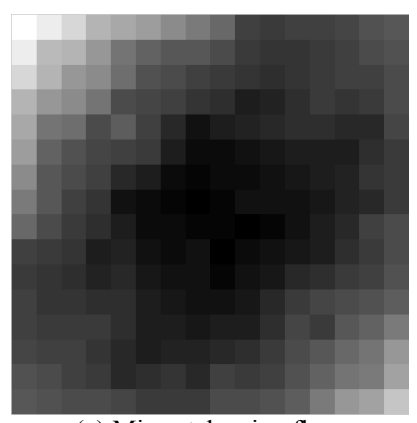

(a) Mismatch using floor

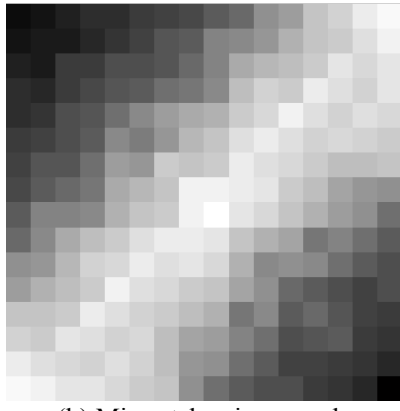

(b) Mismatch using round
Fig. 4. Relationship between $\mathbf{D}_{2 x-l}(\mathbf{p}), \mathbf{D}_{2 x+1}(\mathbf{p})$ and probability of mismatch.

The statistics show that the floor operator offers a low level of mismatch when $\mathbf{D}_{2 x-1}(\mathbf{p})$ and $\mathbf{D}_{2 x+1}(\mathbf{p})$ both approach $\frac{Q S-1}{2}$. In this case, the corresponding $\mathbf{W Z}_{2 \mathrm{x}}(\mathbf{p})$ and $\mathbf{S I}_{2 \mathrm{x}}(\mathbf{p})$ coefficients are expected to be far from the endpoints of the floor operator. Mismatch then increases when $\mathbf{D}_{2 x-1}(\mathbf{p})$ and $\mathbf{D}_{2 x+1}(\mathbf{p})$ approach the endpoints of the interval. Conversely, the round operator follows a different pattern. The probability of mismatch is high when $\mathbf{D}_{2 x-1}(\mathbf{p})$ and $\mathbf{D}_{2 x+l}(\mathbf{p})$ are at the center of the interval and decreases as they approach the endpoints. Fig. 5(a) shows the operator that provides the lowest mismatch between Figs. 4(a)-(b) for the differences $\mathbf{D}_{2 x-1}(\mathbf{p})$ and $\mathbf{D}_{2 x+1}(\mathbf{p})$, using an 'o' for the floor operator and an ' $\mathrm{x}$ ' for the round operator.

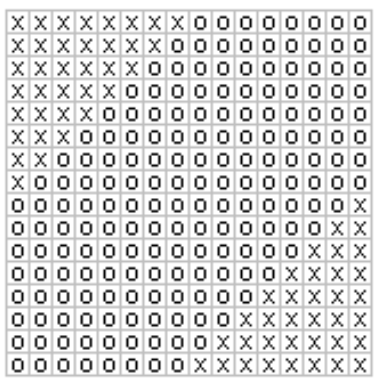

(a) Best operators for Region 1

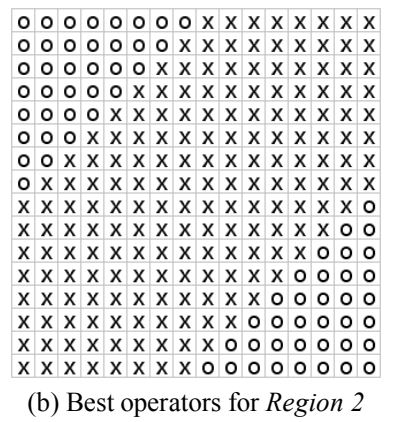

(b) Best operators for Region 2

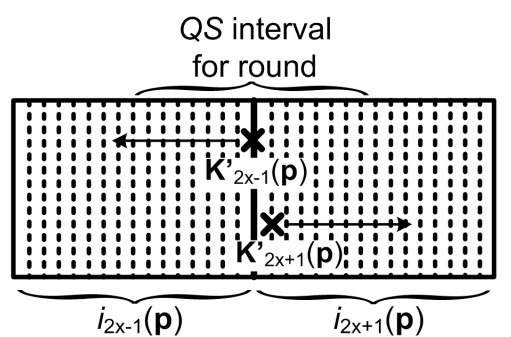

(c) Coefficients $\mathbf{K}_{2 \mathrm{x}-1}(\mathbf{p})$ or $\mathbf{K}_{2 \mathrm{x}+1}(\mathbf{p})$

Fig. 5. (a)-(b)Operators selected for Region 1 and 2; (c) Coefficients $K_{2 x-1}^{\prime}$ or $K_{2 x+1}^{\prime}$

Region 2 includes the locations where the coefficients of the adjacent key frames fall within the adjacent intervals such that $\left|\boldsymbol{i}_{2 x-1}(\mathbf{p})-\boldsymbol{i}_{2 x+1}(\mathbf{p})\right|=1$. These regions can still represent areas of low motion. Yet the statistics collected over Region 2 follow a different relationship with $\mathbf{D}_{2 x-1}(\mathbf{p})$ and $\mathbf{D}_{2 x+1}(\mathbf{p})$ compared to Region 1. Separate statistics, collected over Region 2, show that the round operator offers lower mismatch when say $\mathbf{D}_{2 x-1}(\mathbf{p})$ approaches $Q S$ and $\mathbf{D}_{2 x+1}(\mathbf{p})$ approaches 0 , as shown in Fig. 5(c). This is because the co-located $\mathbf{W Z}_{2 \mathrm{x}}(\mathbf{p})$ and $\mathbf{S I}_{2 \mathrm{x}}(\mathbf{p})$ coefficients are at the centre of the intervals considered by the round operator, which is also illustrated in Fig. 5(c). The level of mismatch increases as $\mathbf{D}_{2 x-1}(\mathbf{p})$ and/or $\mathbf{D}_{2 x+1}(\mathbf{p})$ deviate from the endpoints towards the centre of one of the intervals, following the arrows in Fig 5(c). On the other hand, the floor operator follows an opposite pattern and the best quantization operator that must be chosen for each possible $\mathbf{D}_{2 x-1}(\mathbf{p})$ and $\mathbf{D}_{2 x+1}(\mathbf{p})$ combination is in Fig. 5(b).

Region 3 considers the locations where the adjacent frames are more than one interval apart, such that $\mid \boldsymbol{i}_{2 x-1}(\mathbf{p})$ $\boldsymbol{i}_{2 x+l}(\mathbf{p}) \mid>1$. For such regions of high motion, the $\mathbf{S I}_{2 \mathrm{x}}(\mathbf{p})$ is not likely to predict the $\mathbf{W Z}_{2 \mathrm{x}}(\mathbf{p})$ coefficients well and thus both rounding operators generate wrong soft-input predictions. Due to the weak correlation between the key frames and the probability of mismatch, such regions are quantized using the traditional floor operator at all times, just like the DISCOVER Codec.

Maps like those in Figs. 5(a)-(b) were also generated for the other QP points, and for the AC coefficients, and they were all found to follow a very similar pattern. Hence these maps can be up-sampled and used for all QPs. The AC coefficients are quantized using a dead zone quantizer with double zero interval in [9] where the quantization step size is set by finding the maximum coefficient within the band MaxVal, and divide the dynamic range [-MaxVal, MaxVal) by the number of intervals $2^{L}$ as in [13]:

$$
Q S=\left\lceil\frac{2 \times M a x V a l}{2^{L}}\right\rceil
$$

Fig. 6(a) illustrates the binary map $\mathbf{M}$ used for the DC coefficients of the $51^{\text {st }}$ frame in the Hall monitor, marking as white dots all the locations $\mathbf{p}$ where the round operator is selected. On the other hand, Fig. 6(b) shows the superposition of the locations where the bit-planes of the WZ indices $\mathbf{Q}^{(b)}$ do not match the corresponding bit-planes predicted by the sign of the soft-input predictions $\mathbf{S}^{(b)}$ for all bit-planes $b \in\{1, \ldots, L\}$. Every DC coefficient is quantized using the correct operator indicated in Fig. 6(a), showing that this scheme can improve the soft-input predictions for all bit-planes, compared to Figs. 3(b)-(c). The total WZ bitrate, required to correct the new soft-input values predicted for all the bit-planes of the WZ frames was reduced by $10 \%$ compared to the traditional methods using just the floor operator, incurring only a minor loss of $0.03 \mathrm{~dB}$ in the reconstructed PSNR quality. 


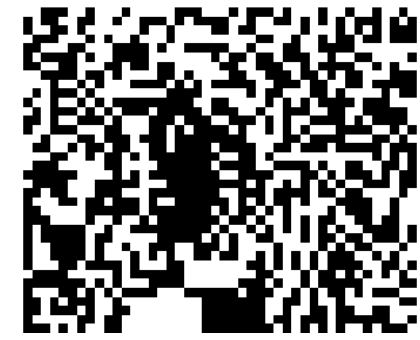

(a) Binary map $\mathbf{M}$

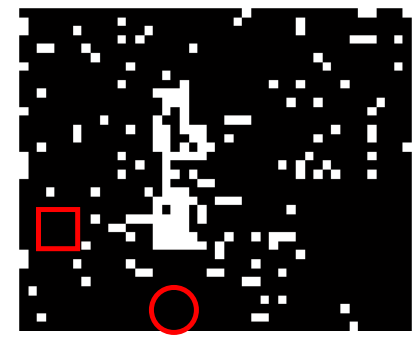

(b) Discrepancy in soft-input values

Fig. 6. Discrepancies using the proposed coding scheme.

\section{EXPERIMENTAL RESULTS}

The Akiyo $(15 \mathrm{~Hz})$ and the Foreman $(30 \mathrm{~Hz})$ sequences having QCIF resolution, and the Hall Monitor $(15 \mathrm{~Hz})$ and Coastguard $(30 \mathrm{~Hz})$ sequences with CIF resolution, were compressed using the proposed Wyner-Ziv video coding architecture. The R-D performances obtained when considering the average luminance component of the WZ frames, and that of the key frames, are shown in Figs. 7(a)(d). To obtain the required R-D points, the DCT coefficients of the WZ frame were quantized using the eight $4 \times 4$ quantization matrices given in [9], which were also used for the DISCOVER project. Conversely, the key frames were Intra coded using H.264/AVC (Main profile) with the Quantization Parameters chosen using an iterative process which stops when the average quality (PSNR) of the WZ frame is similar to the quality of the Intra frames throughout the whole sequence [14]. For comparison purposes, the plot includes the R-D performances of the DISCOVER Codec [14], under the same coding scenario. Both experimental setups consider Gray code representation and are encoded with a GOP size of 2. Furthermore, the plot considers the R$\mathrm{D}$ performances obtained when all the frames are encoded using H.264/AVC Intra coding and H.264/AVC No Motion since only these coding schemes can offer a comparable low encoding capability.

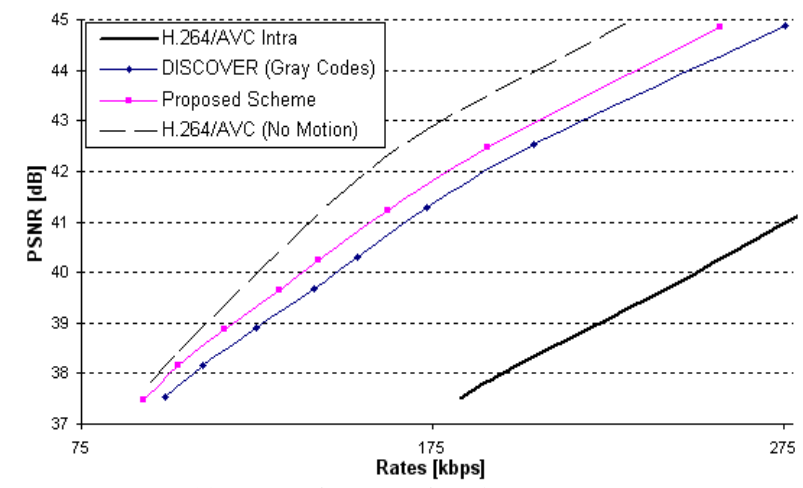

(a) R-D performance for Akiyo sequence.

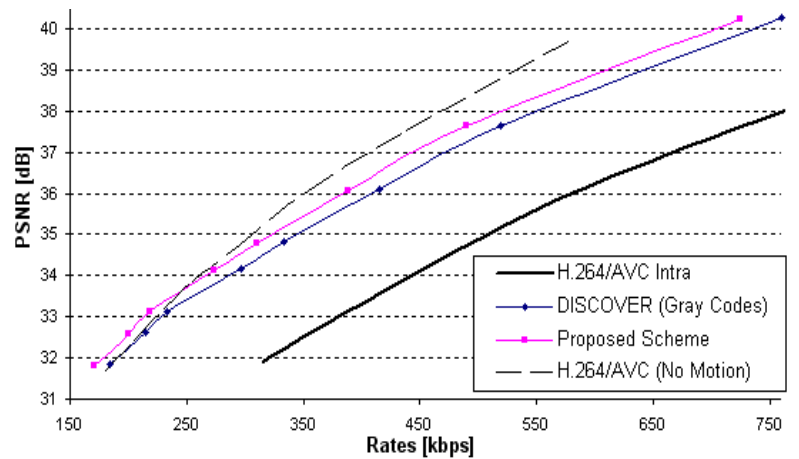

(b) R-D performance for Foreman sequence.

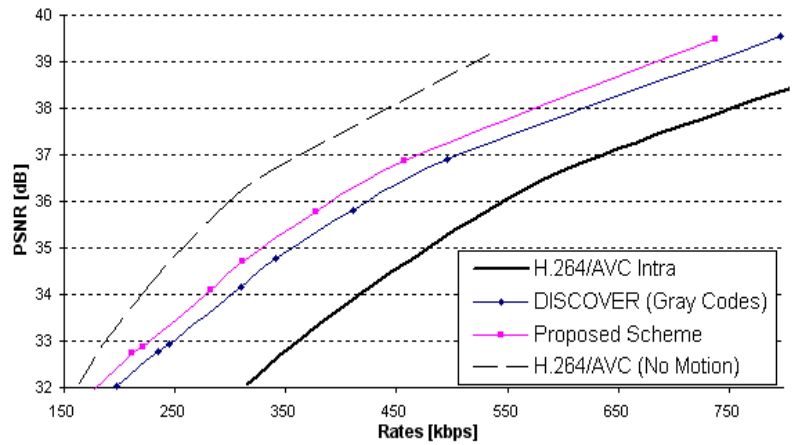

(c) R-D performance for Hall Monitor sequence.

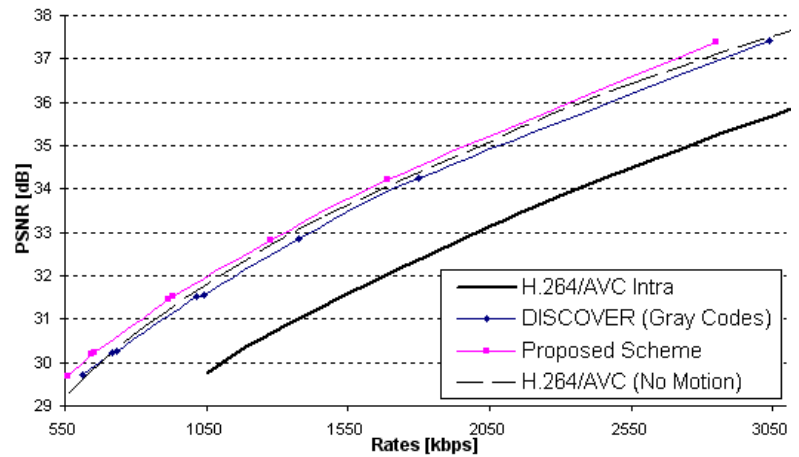

(d) R-D performance for Coastguard sequence.

Fig. 7. R-D performances of the tested sequences.

These figures illustrate that the proposed scheme can improve the accuracy of the soft-input values with little degradation in quality, achieving a significant improvement in R-D performance compared to the DISCOVER Codec [14]. For a good comparative study, the numerical average difference between the two RD-curves was calculated using the Bjøntegaard-Delta (BD) metric [19], obtaining an average BD-PSNR improvement of $0.35 \mathrm{~dB}, 0.37 \mathrm{~dB}, 0.48 \mathrm{~dB}$ and $0.56 \mathrm{~dB}$ for the Foreman, Coastguard, Hall Monitor and Akiyo sequences respectively. Smaller gains were observed for high motion sequences like the Foreman and the Coastguard. In such cases, the large prediction errors in the side information result in incorrect soft-input predictions 
when using either the floor or the round operator. The proposed coding scheme will therefore have a smaller effect on improving the quality of the soft input values, compared to low motion sequences, such as the Hall monitor and Akiyo, where most of the discrepancies are due to small changes in light intensity.

\section{CONCLUSION}

The effect that small variations in light intensity have on the coding performance of traditional DVC architectures has been investigated. An adaptive scheme has been proposed, where the encoder and decoder distinguish between different rounding operators, in an attempt to improve the quality of the soft-input predictions. The experimental results have shown that the proposed methodology can improve the DBPSNR by up to $0.56 \mathrm{~dB}$ compared to the DISCOVER Codec, with a minimal increase in the encoder's complexity.

\section{ACKNOWLEDGMENTS}

The research work disclosed in this publication is partially funded by the Strategic Educational Pathways Scholarship Scheme (Malta). The scholarship is part-financed by the European Union - European Social Fund. (ESF 1.25).

\section{REFERENCES}

[1] T. Wiegand, G. Sullivan, G. Bjøntegaard, and A. Luthra, "Overview of the H.264/AVC video coding standard," IEEE Transactions on Circuits and Systems for Video Technology, vol. 13 , no. 7, pp. 560-576, July 2003.

[2] A. Wyner, and J. Ziv, "The rate-distortion function for source coding with side information at the decoder," IEEE Transaction on Information Theory, vol. 22, no. 1, pp. 1-10, January 1976.

[3] Z. He, L. Cao, and H. Cheng, "Correlation estimation and performance optimization for distributed image compression," in Proc. of SPIE Visual Communications and Image Processing, vol. 6077, pp. 1-8, January 2006.

[4] G. Huchet, Y. Chouinard, D. Wang, and A. Vincent, "Adaptive source representation for distributed video coding," in Proc. of IEEE International Conference on Image Processing, November 2009.

[5] A. L. Becker, and K. M. Leung, "New counting codes for distributed video coding," Computing Research Repository, 2007.

[6] J. J. Micallef, R. A. Farrugia, and C. J. Debono, "Improved Wyner-Ziv video coding efficiency using bit plane prediction," in Proc. of IEEE International Conference on Image Processing, September 2011.

[7] B. Wu, X. Guo, D. Zhao, and F. Wu, "An optimal nonuniform scalar quantizer for distributed video coding," in Proc. of
IEEE International Conference on Multimedia and Expo, July 2006.

[8] W. A. R. Weerakkody, W. A. C. Fernando, M. B. Badem, and A. M. Kondoz, "Nonlinear quantisation for pixel domain distributed video coding," IET Electronic Letters, vol. 45, no. 5, pp. 261-262, February 2009.

[9] C. Brites, J. Ascenso, and F. Pereira, "Improving transform domain Wyner-Ziv video coding performance," in Proc. of IEEE International Conference on Acoustics, Speech, and Signal Processing, May 2006.

[10] C. L. Xiao, L. M. Po, and W. H. Mo, "Adaptive quantization for distributed video coding," in Proc. of Data Compression Conference, March 2011.

[11] C. W. Jung, and L. J. Karam, "BitpLAne SelecTive distributed video coding," in Proc. of Asilomar Conference on Signals, Systems, and Computers, October 2008.

[12] C. W. Jung, and L. J. Karam, "AQT-DVC: Adaptive quantization for transform-domain distributed video coding," in Proc. of IEEE International Conference on Image Processing, September 2010.

[13] X. Artigas, J. Ascenso, M. Dalai, S. Klomp, D. Kubasov and M. Ouaret, "The discover codec: architecture, techniques and evaluation," in Proc. of Picture Coding Symposium, November 2007.

[14] The Discover Codec [online]. Available: www.discoverdvc.org.

[15] D. Varodayan, A. Aaron, and B. Girod, "Rate-adaptive codes for distributed source coding," EURASIP Signal Processing Journal, vol. 86, no. 11, November 2006.

[16] J. Ascenso, C. Brites, and F. Pereira, "Improving frame interpolation with spatial motion smoothing for pixel domain distributed video coding," in Proc. of EURASIP Conference on Speech, and Image Processing, July 2005.

[17] C. Brites, and F. Pereira, "Correlation noise modeling for efficient pixel and transform domain Wyner-Ziv video coding," IEEE Transactions on Circuits and Systems for Video Technology, vol. 18, no. 9, September 2008.

[18] S. Cheng, and Z. Xiong, "Successive refinement for the Wyner-Ziv problem and layered code design," IEEE Transactions on Signal Processing, vol. 53, no. 8, pp. 3269-3281, August 2005.

[19] D. Kubasov, J. Nayak, and C. Guillemot, "Optimal reconstruction in Wyner-Ziv video coding with multiple side information," in Proc. of IEEE International Workshop on Multimedia Signal Processing, October 2007.

[20] G. Bjøntegaard, "Calculation of average PSNR differences between RD curves," in VCEG Meeting, April 2001. 